139. A. Skita und H. Rolfes: Über Cyclohexylamine.

[Aus dem Chem. Institut der Universität Freiburg i. Br.]

(Eingegangen am 3. Mai 1920.)

Adolf von Baeyer hatte im Jahre 1893 das Cyclohexylamin durch Reduktion des »Pimelinketon-Oxims" hergestellt") und diese Base durch das salzsaure Salz, die Acetyl- und Benzoylverbindung, den Harnstoff und Thio-harnstoff charakterisiert; später hat noch N. Kish$\left.n e r^{2}\right)$ das Cyclohexyl-hydrazin durch Einwirkung von Hydrazin auf Keto-hexamethylen hergestellt. Obwohl das Cyclohexylamin - das inzwischen von Markownikoff auch durch Reduktion des Nitrohexamethylens aus dem kaukasischen Erdöl erhalten wurde ${ }^{3}$ - durch die katalytische Reduktion nach Sabatier und Senderens direkt aus dem Anilin ${ }^{4}$ ) hergestellt werden konnte, blieb die Chemie des hexahydrierten Anilins doch im wesentlichen auf die genannten Derivate beschränkt.

Da uns im Anschluß an die allgemein anwendbare Umwandlung der Aniline in Cyclohexylamine die Herstellung dieser cyclischen Basen leicht möglich war ${ }^{5}$ ), haben wir versucht, die Chemie der hydrierten Aniline durch die nachfolgend beschriebenen Versuche etwas zu erweitern.

Es zeigte sich, daß bei der Destillation des $N, \boldsymbol{N}$-Di-cyclohexylthioharnstoffs (I.), erhalten aus Schwefelkohlenstoff und Cyclohexylamin, mit glasiger Phosphorsäure eine stechend riechende Flüssigkeit rom Sdp. $219^{\circ}$ das Cyclohexyl-isothiocyanat (II.) entstand:

$\mathrm{CS}-\mathrm{NH} . \mathrm{C}_{6} \mathrm{H}_{11}(\mathrm{I}) \rightarrow \mathrm{C}_{6} \mathrm{H}_{11} . \mathrm{N}: \mathrm{C}: \mathrm{S}(\mathrm{II}$. $)+\mathrm{C}_{6} \mathrm{H}_{11} \cdot \mathrm{NH}_{2}$.

Dieser Körper wurde in besseren Ausbeuten nach der Hofmannschen Senföl Synthese ${ }^{b}$ ) durch Einwirkung von $\mathrm{Hg} \mathrm{Cl}_{2}$ auf di-cyclohexyl-dithiocarbaminsaures Ammonium (III.), das erste Einwirkungsprodukt von $\mathrm{CS}_{2}$ auf Cyclohexylamin, erhalten:

$\mathrm{CS}<\mathrm{S} . \mathrm{NH}_{3} \cdot \mathrm{C}_{6} \mathrm{H}_{11} \mathrm{H}_{11} \quad($ Ill. $) \rightarrow \mathrm{C}_{6} \mathrm{H}_{11} \cdot \mathrm{N}: \mathrm{C}: \mathrm{S}+\mathrm{H}_{2} \mathrm{~S}+\mathrm{C}_{6} \mathrm{H}_{11} \cdot \mathrm{NH}_{2}$.

Das Cyclohexyl-isothiocyanat, der erste Vertreter der hydroaromatischen Senföle, ist so wie die aliphatischen und aromatischen Senföle sehr reaktionsfähig und bildet besonders glatt mit Alkoholen und Phenolen Thio-urethane (IV.) und mit Ammoniak und

1) A. 278, 88 [1893]. 2) K. 43, 577; C. 1911, II 362.

ग) A. 302, 1 [1898]. ${ }^{4}$ ) C. r. 138, 457; C. 1904, I 884.

5) A. Skita und W. Berendt, B. 52, 1519 [1919],

E) B. 8, 108 [1875]; 23, 282 [1890]; B. 29, Ref. 651 [1896]. 
Aminen Thio-harnstoffe (V.). Durch Einwirkung von Anilin auf das Cyclohexylsenföl bildete sich der von $A$. von Baeyer auf anderem Wege erhaltene $N$-Phenyl- $N^{\prime}$-cyclohexyl-thioharnstoff ${ }^{1}$, bei der Einwirkung von Cyclohexylamin der $N, N^{\prime}$-Di-cyclohexylthioharnstoff (I.).

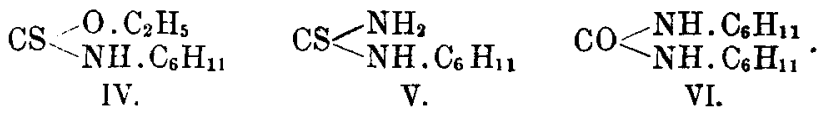

Aus Phosgen und Cyclohexylamin entstand der $N, N^{\prime}$ - Di-cyclohexyl-harnstoff (VI.), der, mit $\mathrm{P}_{2} \mathrm{O}_{5}$ destilliert, unter Abspaltung von Cyclohexylamin das Cyclohexyl-isocyanat, $\mathrm{C}_{6} \mathrm{H}_{11} . \mathrm{N}: \mathrm{C}: \mathrm{O}$ eine Flüssigkeit vom Sdp. $176^{\circ}$ von stechendem, die Augen zu Tränen reizendem Geruch bildete.

Dieser Körper, der erste Vertreter der Klasse der hydrocyclischen Isocyanate, ist von großer Reaktionsfähigkeit, besonders mit Alkoholen und Phenolen, mit welchen er Urethane (VII.) und mit Aminen, Ammoniak und Anilinen, mit welchen er Harnst offe (VILI.) liefert. So entstand .durch Einwirkung von Anilin auf das Cyclohexyl-isocayanat der von A. von Baeyer auf anderm Wege erhaltene $N$-Phen yl- $N^{\prime}$-cyclohexyl-harnstoff (IX.), durch Einwirkung von Cyclohexylamin der $N, N^{\prime}$ - Di-cyclohexyl-harnstoff (VI.).

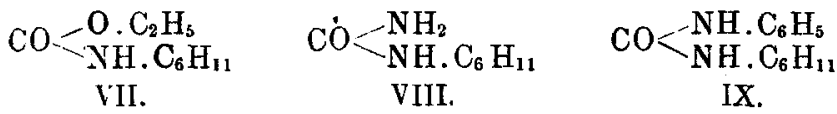

Die Alkylierung des Cyclohexylamins mit Dimethylsulfat gab im wesentlichen $N$-Methyl-cyclobexylamin, die mittels Formaldehyds und Ameisensäure $\left.{ }^{2}\right) N$-Dimethyl-cyclobexylamin als Hauptprodukt. Die beiden $\mathrm{N}$-alkylierten Basen sind über die Acetyl- oder Benzoylverbindung des $N$-Methyl-cyclohexylamins leicht zu trennen und in reinem Zustande zu erhalten; sie sind identisch mit den durch katalytische Hydrierung des $\mathrm{N}$-Methyl- und $\mathrm{N}$-Dimethyl-anilins erzeugten Basen ${ }^{3}$, mit denen sie außer durch ihren Siedepunkt noch durcb Salze und Nitrosoverbindung, sowie durch das Trimethylammoniumjodid identifiziert wurden. In gleicher Weise stimmen die durch Äthylierung mit Jodäthyl erhaltenen $N$-Äthyl-cyclohexylamine mit den durch direkte HSdrierung des $\boldsymbol{N}$-Mono- und -Diäthylanilins erhaltenen Basen $^{2}$ ) überein. Das $\boldsymbol{N}$-Äthyl-cyclohex ylamin bildet sich aus berechneten Mengen Cyclohexylamin und Jodäthyl quantitativ in Form seines jodwasserstoffsauren Salzes. Das $N$-Diäthyl-cy cloh ex yla min vom Sdp.
3) loc. cit.
3) K. HeB, B. 48, 1888 [1915].
3) C. r. 138,1258 (C. 1904, II 105); B. 62, 1527 [1914]. 
$193^{\circ}$ entsteht beim Kochen des Cyclohexylamins mit berechneter Menge Jodäthyl und Alkali; beides in quantitativer Ausbeute.

Von den Derivaten der $\mathbf{N}$-alkylierten Cyclohexylamine ist die Nitroso-Verbindung bemerkenswert, welche durch Einwirkung vou Nitrosylchlorid auf $N$-Dimethyl-cyclohexylamin in ätherischer Lösung entsteht, ein gelbliches Öl vom Sdp. $121^{\circ}$ bei $12 \mathrm{~mm}$. Bei der Reduktion dieser Nitroso-Verbindung mit Zinn und Salzsäure entstand eine Base, die durch ihren Sdp. $145^{\circ}$ und durch ihre Derivate, besonders durch ihre bei $76^{\circ}$ schmelzende Benzoylverbindung sich zweifellos als $N$-Methyl-cyclohexylamin erwies:

$\mathrm{C}_{6} \mathrm{H}_{11} \cdot \mathrm{N}\left(\mathrm{CH}_{3}\right)_{2}+\mathrm{NOCl} \rightarrow \mathrm{C}_{6} \mathrm{H}_{11} \cdot \mathrm{N}(\mathrm{NO}) \cdot \mathrm{CH}_{3} \rightarrow \mathrm{C}_{6} \mathrm{H}_{11} \cdot \mathrm{NH} . \mathrm{CH}_{3}$.

Fs war also das tertiäre Alkyl durch die NO-Gruppe verändert worden. Dasselbe $N$-Nitroso- $N$-methyl-cyclobexylamin entstand bei der Einwirkung von Nitrosylchlorid auf $N$-Methyl-cyclohexylamin in ätherischer Lösung:

$$
\begin{aligned}
2 \mathrm{C}_{6} \mathrm{H}_{11} \cdot \mathrm{NH} \cdot \mathrm{CH}_{3}+\mathrm{NOCl}= & \mathrm{C}_{6} \mathrm{H}_{11} \cdot \mathrm{N}(\mathrm{NO}) \cdot \mathrm{CH}_{3} \\
& +\mathrm{C}_{6} \mathrm{H}_{11} \cdot \mathrm{NH} \cdot \mathrm{CH}_{3}, \mathrm{HCl} .
\end{aligned}
$$

Wir sind noch einem zweiten Fall des Ersatzes des tertiären Alkyls durch die Nitroso-Gruppe begegnet bei der Einwirkung von Nitrosylchlorid auf $N$-Methyl-dicyclohexylamin, eine Base, deren Herstellung kürzlich beschrieben wurde ${ }^{1}$ ):

$$
\mathrm{C}_{6} \mathrm{H}_{11} \cdot \mathrm{N}\left(\mathrm{CH}_{3}\right) \cdot \mathrm{C}_{6} \mathrm{H}_{11}+\mathrm{NOCl} \rightarrow \mathrm{C}_{6} \mathrm{H}_{11} \cdot \mathrm{N}(\mathrm{NO}) \cdot \mathrm{C}_{6} \mathrm{H}_{11} \text {. }
$$

Hierbei entstand das $N$-Nitroso-dicyclohexylamin, das aus Äther in Krystallen rom Schmp. $105^{\circ}$ erbalten wurde. Bei der Reduktion dieser Nitroso-Verbindung wurde das Dicyclohexylamin erhalten, eine Base, die sowohl durch direkte Hydrierung des Diphenylamins, wie auch als Nebenprodukt bei der katalytischen AnilinHydrierung entsteht, und die mit Nitrosylchlorid behandelt, dasselbe $N$-Nitroso-dicyclohexylamin lieferte:

$$
\begin{aligned}
2 \mathrm{C}_{6} \mathrm{H}_{11} \cdot \mathrm{NH} . \mathrm{C}_{6} \mathrm{H}_{11}+\mathrm{NOCl}= & \mathrm{C}_{6} \mathrm{H}_{11} \cdot \mathrm{N}(\mathrm{NO}) \cdot \mathrm{C}_{6} \mathrm{H}_{11} \\
& +\mathrm{C}_{6} \mathrm{H}_{11} \cdot \mathrm{NH} . \mathrm{C}_{6} \mathrm{H}_{11}, \mathrm{HCl} .
\end{aligned}
$$

Während die katalytische Wasserstoff-Anlagerung an den Benzolring bei den $N$-alkylierten Anilinen ebenso leicht wie bei den Anilinen selbst erfolgt, haben wir an dem Beispiel des Acetanilids festgestellt, daB durch die Acetylierung der Aminogruppe die Hydrierung des Benzolringes wesentlich erschwert wird und bei den geringen Platin-Konzentrationen, bei denen die Anilin-Hydrierungen ausgeführt wurden, überhaupt nicht durchgeführt werden konnte $\left.{ }^{2}\right)$. Dagegen ge-

1) A. Skita und W. Berendt, B. 52, 1522 [1919].

2) Diplomarbeit V. H. Kah, Karlsruhe i. B. (1912). 
lang die Umwandlung des Antifebrins zu dem Hexahydro-antifebrin schließlich schon in 45 Minuten, als die Hydrierung in gröBerer Platin-Konzentration bei ca. $80^{\circ}$ vorgenommen wurde.

Auch die acetylierten Toluidine gaben auf diese Weise glatt die bisher noch nicht bekannten acetylierten 2-, 3- und 4-Methylcyclohexylamine.

Ein wesentlicher Unterschied $\mathrm{zwischen}$ der Hydrierung der acetylierten und der freien Aniline bestand auch darin, daB sich im ersten Falle keine Spur der Dicyclohexylamine bildete, welche bei der katalytischen Anilin-Hydrierung oft in sehr wesentlicher Menge auftreten $^{1}$ ).

Als naher Verwandter des Acetanilids wurde sodann das Phenacetin ( $p$ - Äthoxy-acetanilid) der katalytischen Platin Hydrierung unterworfen. Unter besonders leichter Wasserstoff-Aufnahime wurden hierbei an Stelle der erwarteten 3 Mol. 4 Mol. Wasserstoff aufgenommen, und das Reaktionsprodukt erwies sich dementsprechend als Hexahydro-acetanilid:

$$
\mathrm{C}_{6} \mathrm{H}_{4}\left(\mathrm{OC}_{2} \mathrm{H}_{5}\right)\left(\mathrm{NH} . \mathrm{COCH}_{3}\right) \rightarrow \mathrm{C}_{6} \mathrm{H}_{11} \cdot \mathrm{NH} . \mathrm{COCH}_{3} \text {. }
$$

Die Erschwerung der Wasserstoff-Aufnahme durch die Acetylierung der A minogruppe bedeutet also noch keine Festigung einer gleichzeitig anwesenden Hydroxylgruppe. Auch kann die Eigenschaft, leicht reduziert zu werden, der eingeführten Alkoxygruppe als solcher nicht zukommen, da unter denselben Bedingungen sowohl das Anisol als auch das Phenetol in den korrespondierenden hydrocyclischen Äther ${ }^{2}$ ) umgewandelt werden konnte:

$$
\left\{\begin{array}{ccl}
\mathrm{C}_{6} \mathrm{H}_{5} \cdot \mathrm{O} \cdot \mathrm{CH}_{3} & 3 \mathrm{H}_{2} \rightarrow & \mathrm{C}_{6} \mathrm{H}_{11} \cdot \mathrm{O} \cdot \mathrm{CH}_{3} \\
\text { Anisol } & & \text { Cyclohexyl-methyl-äther } \\
\mathrm{C}_{6} \mathrm{H}_{5} \cdot 0 . \mathrm{C}_{2} \mathrm{H}_{5} & 3 \mathrm{H}_{2} \rightarrow & \mathrm{C}_{6} \mathrm{H}_{12} \cdot \mathrm{O} \cdot \mathrm{C}_{2} \mathrm{H}_{5} \\
\text { Phenetol } & & \text { Cyclohexyl-äthyl-äther. }
\end{array}\right.
$$

Die leichte Reduktion der OH-Gruppe, sowie der Benzol-Doppelbindungen bei den Amino-phenolen muß also in Zustandsänderungen des Benzolkerns zu suchen sein, welche durch den Eintritt der $\mathrm{OH}$-Gruppe in das Molekül des Anilins hervorgerufen worden ist, und die wir als Auflockerung des Benzolkerns bezeichnen, ein Zustand, in welchem der Benzolkern den Wasserstoff leichter als in nicht substituiertem Zustand aufnimmt, und in welchem die Benzol-Doppelbindungen einen mehr aliphatischen Charakter angenommen haben. In Übereinstimmung damit wurde sowohl das

1) B. 52, 1522 [1919].

2) L. Brurel, Bt. [3] 33, 272 (C. 1905, I 1014). 
Anisidin, wie auch das Phenetidin glatt zu Cyclobexylamin reduziert und dieselbe Erscheinung auch bei der katalytischen Hydrierung der drei A mino-phenole beobachtet, womit auch bewiesen war, dab die OH-Gruppe nicht nur in der $p$-Stellung, sondern auch in der $o$ - und $m$-Stellung des Anilins die Lockerung des Benzolkerns bewirkt hat. Auch in diesen Fällen verlief die katalytische Reduktion sehr glatt bis zu dem Cyclohexylamin:

$\mathrm{C}_{6} \mathrm{H}_{4}\left(\mathrm{OC}_{2} \mathrm{H}_{5}\right)\left(\mathrm{NH}_{2}\right) \rightarrow \mathrm{C}_{6} \mathrm{H}_{11} \cdot \mathrm{NH}_{2}$;

$$
\mathrm{C}_{6} \mathrm{H}_{4}(\mathrm{OH})\left(\mathrm{NH}_{2}\right) \rightarrow \mathrm{C}_{6} \mathrm{H}_{12} \cdot \mathrm{NH}_{3} \text {. }
$$

Etwas anders als $p$ - und $m$-Amino-phenol verbielt sich allerdings das o-Amino- bezw. o-Nitro-phenol, welches nur zu etwa $85 \%$ Cyclohexylamin lieferte, dagegen etwa $15 \%$ einer Verbindung ergab, die aus der alkoholisch gemachten Reaktionsflüssigkeit nach dem Cyclohexylamin mit Wasserdämpfen destillierte und aus Petroläther in Nadeln vom Schmp. 103-10 $4^{0}$ erhalten wurde. Die Analyse zeigte, daB hier ein bimolekulares Kondensationsprodukt in Gestalt eines hydroaromatischen Oxazins:

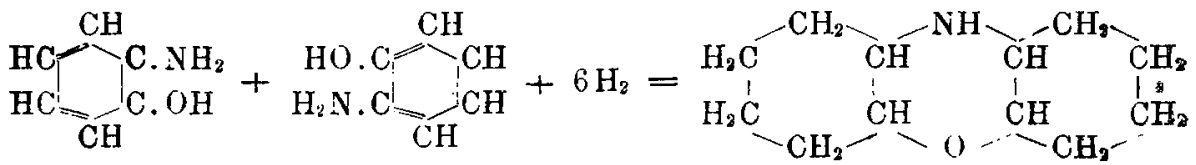

vorlag, das unter Ammoniak- und Wasser-Abspaltung analog den aromatischen Oxazinen entstanden war. Die Imidogruppe wurde durch die Benzoylierung nachgewiesen und die Benzoyl-Verbindungdes Dodekabydro-phenoxazins in Pyramiden vom Scbmp. 160-1610 erbalten.

Die Badische Anilin- und Soda-Fabrik hatte uns zur Ausführung dieser Versuche Cyclohexylamin zur Verfügung gestellt, wofür wir ihr unsern besten Dank aussprechen. Der Wissenschaftlichen Gesellschaft in Freiburg i. Br. sind wir für eine Unterstützung zu Dank verpflichtet, die es uns ermöglichte, trotz des teuren Platinpreises diese Arbeit durchzuführen. Schließlich danken wir noch dem Hrn. Dr. P. Stukart, sowie Hrn. H. Häuber für ihre tätige Mitarbeit bei diesen Versuchen.

Versuche, gemeinsam mit den HHrn. cand. chem. Karl Hils und Gustav Kirchboff.

I. Thio-harnstoffe und Senföl des Cyclohexylamins.

1. Dicyclobexyl-dithiocarbaminsaures Ammoniak (III.).

In $5 \mathrm{~g}$ Cyclohexylamin wurden unter Eiskühlung $2 \mathrm{~g}$ Schwefelkohlenstoff eingetropft; das unmittelbar gebildete feste Reaktionsprodukt gab aus Alkohol Krystalle vom Schmp. 160 . 
0.1623 g Sbst.: $0.2769 \mathrm{~g} \mathrm{BaSO}$.

$\mathrm{C}_{13} \mathrm{H}_{26} \mathrm{~N}_{2} \mathrm{~S}_{2}$ (274). Ber. S 23.36. Gef. S 23.44.

2. $N, N^{\prime}$-Di-cyclohexyl-thioharnstoff (I.).

$10 \mathrm{~g}$ Cyclohexylamin, in $25 \mathrm{~g}$ Alkohol gelöst, wurden mit $10 \mathrm{~g}$ Schwefelkohlenstoff unter Zugabe eines Körnchens Kaliumbydroxyd bis zum Auibören der Schwefel wasserstoff-Entwicklung erwärmt; nach dem Abdestillieren des Alkohols wurde der Thiobarnstoff in Würfeln vom Schmp. $180-181^{\circ}$ (aus Weingeist) erhalten.

$0.1598 \mathrm{~g}$ Sbst.: $0.1564 \mathrm{~g} \mathrm{BaSO}$.

$\mathrm{C}_{13} \mathrm{H}_{24} \mathrm{~N}_{2} \mathrm{~S}$ (240). Ber. S 13.36. Gef. S 13.45.

\section{Cyclobexylsenföl (II.).}

Bei der Destillation des $N, N^{\prime}$-Di-cyclohexpl-thioharnstoffes mit glasiger Phosphorsäure wurde eine äußerst stechend nach Senföl riechende Flüssigkeit erbalten; in besseren Ausbeuten entstand diese Substanz, als zu $10 \mathrm{~g}$ Cyclohexylamin in absolutem Äther gelöst $3.8 \mathrm{~g}$ $\mathrm{CS}_{3}$ zugefügt und nach dem Abdampien des Äthers das dithiocarbaminsaure Ammonium mit $13.5 \mathrm{~g}$ Sublimat und $200 \mathrm{ccm}$ Wasser erbitzt wurde. Das Senföl destillierte hierbei am absteigenden Kühler ab, wurde ausgeäthert und zeigte nach dem Trocknen den Sdp. $219^{\circ}$ bei $746 \mathrm{~mm}$. Ausbeute $9 \mathrm{~g}$.

0.1213 g Sbst.: $10.8 \mathrm{ccm} \mathrm{N}\left(16^{0}, 742 \mathrm{~mm}\right)$. $-0.1537 \mathrm{~g}$ Sbst.: $0.2563 \mathrm{~g} \mathrm{BaSO}_{4}$. $\mathrm{C}_{7} \mathrm{H}_{11}$ NS (141). Ber. N 10.08, S 22.70.

Gef. $=9.93,-22.90$.

4. Cyclobexyl-thioharnstoff (V.).

Cyclohexylsenföl wurde unter Erwärmen mit trocknem Ammoniak gesättigt; nach einigen Stunden krystallisierten Säulen rom Schmp. 161-162 (aus Alkohol).

0.0990 g Sbst.: $15.5 \mathrm{cem} \mathrm{N}\left(16^{0}, 742 \mathrm{~mm}\right)$.

$\mathrm{C}_{7} \mathrm{H}_{14} \mathrm{~N}_{2} \mathrm{~S}$ (158). Ber. N 17.72. Gef. N 17.73.

5. N-Phenyl-N'-cyclohexyl-thioharnstoff, $\mathrm{CS}\left(\mathrm{NH} . \mathrm{C}_{6} \mathrm{H}_{11}\right)\left(\mathrm{NH} . \mathrm{C}_{6} \mathrm{H}_{5}\right)$.

Äquimolekulare Mengen Cyclohexylsenföl und Anilin erstarren beim Schütteln rasch zu Krystallen, die, aus Alkohol umgelöst, bei 150-1510 schmelzen. Derselbe Körper entsteht bei Einwirkung von 2,7 g Phenylsenföl auf $1.8 \mathrm{~g}$ Cyclohexylamin unter lebhafter Erwärmung.

$0.1450 \mathrm{~g}$ Sbst.: $0.1470 \mathrm{~g} \mathrm{BaSO}_{4}$.

$\mathrm{C}_{13} \mathrm{H}_{18} \mathrm{~N}_{2} \mathrm{~S}$ (234). Ber. S 13.70. Gef. S 13.92. 


\section{Cyclobexyl-thiourethan (IV.)}

bildet sich bei dreistündigem Erhitzen von $2 \mathrm{~g}$ Cyclohexylsenföl und $5 \mathrm{~g}$ absolutem Äthylalkohol im Rohr auf $100-110^{\circ}$. Derselbe Körper bildet sich auch bei längerem Stehen einer Auflösung von Cyclohexylsenföl in alkoholischer Kalilauge. Krystalle aus absolutem Alkohol rom Schmp. $45-46^{\circ}$.

$0.2095 \mathrm{~g}$ Sbst.: $14.10 \mathrm{~cm} \mathrm{~N}\left(16^{\circ}, 743 \mathrm{~mm}\right)$.

$\mathrm{C}_{9} \mathrm{H}_{17} \mathrm{ONS}$ (187). Ber. N 7.49. Gef. N 7.62.

II. Harnstoffe und Isocyanat des Cyclohexylamins.

I. $N, N^{\prime}$ - Di-cyclohexyl-harnstoff (VI.)

bildet sich leicht beim Einleiten von Phosgen in Cyclohexylamin, wobei sich als Nebenprodukt salzsaures Cyclohexylamin abscheidet. Die Base wird mit Alkali abgetrennt und der Harnstoff aus Alkohol umkrystallisiert. Schmp: $229-230^{\circ}$.

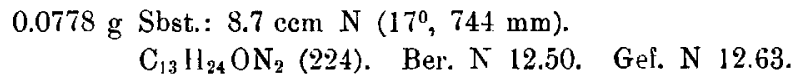

2. Cyclohexyl-isocyanat, $\mathrm{C}_{6} \mathrm{H}_{11} \cdot \mathrm{N}: \mathrm{C}: \mathrm{O}$.

$20 \mathrm{~g}$ Cyclohex flamin fließen unter Eiskühlung in $100 \mathrm{~g}$ 20-proz. Phosgenlösung in Toluol ein. Das nicht umgesetzte Cyclohexylamin wird aus seinem salzsauren Salz durch schwaches Erwärmen mit Alkali freigemacht, vom Di-cyclohexyl-harnstoff abgetrennt, der mit Phosphorsäure-anhydrid gemischt und trocken destilliert wird. Hierbei geht ein stark zu Tränen reizendes öl über vom Sdp. $175^{\circ}$ bei $750 \mathrm{~mm}$.

0.1302 g Sbst.: $13.1 \mathrm{cem} \mathrm{N}\left(19^{\circ}, 750 \mathrm{~mm}\right)$,

$\mathrm{C}_{7} \mathrm{H}_{11} \mathrm{ON}$ (125). Ber. $\mathrm{N} 11.20$. Gef. N 11.35.

\section{Cyclohexpl-harnstoff (VIII.).}

Beim Einleiten von gasförmigem Ammoniak in Cyclobexyl-isocyanat entsteht sofort ein weißer Niederschlag, der aus Alkohol Krystalle rom Schmp. $184^{\circ}$ ergab.

0.0869 g Sbst.: $15.3 \mathrm{ccm} \mathrm{N}\left(17^{\circ}, 742 \mathrm{~mm}\right)$.

$\mathrm{C}_{7} \mathrm{H}_{14} \mathrm{ON}_{2}$ (142). Ber. N 19.72. Gef. $\perp 19.85$.

4. $N$-Phenyl- $N^{\prime}$ - cyclohexyl-harnstoff (IX.)

entsteht sofort in Form eines Krystallbreies beim Mischen äquimolekularer Mengen Cyclohexyl-isocyanat mit Anilin. Weiße Nadeln aus Aceton rom Schmp. $182^{\circ}$.

$0.1568 \mathrm{~g}$ Sbst.: $18.0 \mathrm{ccm} \mathrm{N}\left(16.5^{0}, 742 \mathrm{~mm}\right)$.

$\mathrm{C}_{13} \mathrm{H}_{18} \mathrm{ON}_{2}$ (218). Ber. $\mathrm{N} 12.85$. Gef. $\mathrm{N} 12.98$. 
Dieselbe Substanz bildet sich beim Versetzen von Cyclohexylamin mit Phenyl-isocyanat.

\section{Cyclohexyl-urethan (VII.)}

bildet sich bei 3-stündigem Erwärmen ron $2 \mathrm{~g}$ Cyclohexyl-isocyanat und $5 \mathrm{~g}$ absolutem Alkohol im Rohr auf $110^{\circ}$. Krystalle aus Weingeist vom Schmp. $57^{\circ}$.

$0.1689 \mathrm{~g}$ Sbst : $12.5 \mathrm{ccm} \mathrm{N}\left(18^{0}, 742 \mathrm{~mm}\right)$.

$\mathrm{C}_{9} \mathrm{H}_{17} \mathrm{O}_{2} \mathrm{~N}$ (171). Ber. N 8.19. Gef. N 8.31 .

III. Die Alkylierung des Cyclobexylamins.

1. Mit Dimethylsulfat zu $N$-Methyl-cyclohexylamin.

$10 \mathrm{~g}$ Cyclohexylamin, mit $6 \mathrm{~g}$ Dimethylsulfat in $50 \mathrm{ccm}$ Äther versetzt, gaben beim Erwärmen einen Niederscblag von methylschwefelsaurem Cyclohexylamin. Nadeln vom Schmp. 1030 (aus Eisessig + Äther).

$.0 .1573 \mathrm{~g}$ Sbst.: $0.1750 \mathrm{~g} \mathrm{BaSO}$.

$\mathrm{C}_{7} \mathrm{H}_{17} \mathrm{O}_{4} \mathrm{NS}$ (211). Ber. S 15.1. Gef. S 15.2.

Nach dem Abfiltrieren des Salzes wurde der Äther abgedampft und zur Abtrennung von nebenbei entstandenem $N$-Dimethyl-cyclohexylamin mit Essigsäure-anhydrid auf dem Wasserbade erwärmt.

Acetyl-N-Methyl-cyclobexylamin: Öl rom Sdp. $249^{\circ}$ bei $740 \mathrm{~mm}$.

$03547 \mathrm{~g}$ Sbst.: $29.4 \mathrm{~cm} \mathrm{~N}\left(21^{0}, 742 \mathrm{~mm}\right)$.

$\mathrm{C}_{9} \mathrm{H}_{17} \mathrm{ON}(155)$. Ber. $\mathrm{N} 9.0$. Gef. N 9.1.

Das Benzoyl- $N$-methyl-cyclohexylamin krystallisiert aus Alkohol in Nadeln rom Scbmp. $76^{\circ}$.

Die aus der Acetyl- bezw. Benzoyl-Verbindung mit alkoholischem Kali verseifte Base siedete bei $147^{\circ}$ und ist mit dem durch die kata-

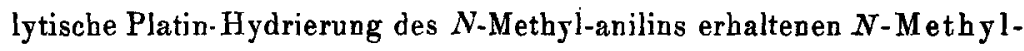
cyclobexylamin') identisch, was außer durch den Siedepunkt noch durch die Identität der nacbfolgend beschriebenen Derivate hervorgebt.

$$
\text { Nitroso-N-Methyl-cyclobexylamin }
$$

entsteht durch Behandlung von $5 \mathrm{~g} \mathrm{~N}$-Methyl-cyclohexylamin in ätherischer Lösung mit Nitrosylchlorid bis zur Neutralisation. Das salzsaure Salz des $N$-Methyl-cyclobexylamins sebied sich in Nadeln rom Schmp. $176^{\circ}$ ab, wïhrend nach dem Abdampfen des Äthers eine gelbliche Flüssigkeit rom Sdp. $121^{\circ}$ bei $12 \mathrm{~mm}$ hinterblieb.

0.2433 g Sbst.: $43.8 \mathrm{ccm} \mathrm{N}\left(21^{\circ}, 740 \mathrm{~mm}\right)$.

$\mathrm{C}_{7} \mathrm{H}_{14} \mathrm{ON}_{3}$ (142). Ber. N 19.7. Gef. N 19.8.

1) B. 52, 1527 [1919]. 
Das Pikrat entstebt aus N-Methyl-eyolohexylamin und alkoholischer Pikrinsäure; aus Alkohol gelbe Krystalle vom Schmp. $170^{\circ}$.

$0.2410 \mathrm{~g}$ Sbst.: $34.4 \mathrm{ccm} \mathrm{N}^{\prime}\left(19^{0}, 744 \mathrm{~mm}\right)$.

$\mathrm{C}_{13} \mathrm{H}_{18} \mathrm{O}_{7} \mathrm{~N}_{4}$ (342). Ber. $\mathrm{N}$ 16.3. Gef. $\mathrm{N} 16.0$.

2. Mit Formaldehyd und Ameisensäure zu $N$-Dimethylcyclohex ylamin.

$10 \mathrm{~g}$ Cyclobexylamin, $20 \mathrm{ccm}$ Wasser, $18 \mathrm{~g} \mathrm{40-proz.} \mathrm{Formaldehyd}$ und $10 \mathrm{~g}$ Ameisensäure wurden im Rohr 4 Stdn. auf $120^{\circ}$ erhitzt. Neben wenig $N$-Methyl-cyclohexylamin, das durch seine bei $76^{\circ}$ scbmelzende Benzoyl-Verbindung abgeschieden wurde, bildet sich zu $80 \%$ der Theorie das bei $165^{\circ}$ siedende $N$-Dimethyl-cyclohexylamin:

$$
\begin{aligned}
\mathrm{C}_{6} \mathrm{H}_{11} \cdot \mathrm{NH}_{2}+2 \mathrm{H} \cdot \overline{\mathrm{COOH}} & +2 \mathrm{H} \cdot \mathrm{C} \mid \overline{\mathrm{HO}} \overline{1} \\
& =\mathrm{C}_{6} \mathrm{H}_{11} \cdot \mathrm{N}\left(\mathrm{CH}_{3}\right)_{2}+2 \mathrm{CO}_{3}+\mathrm{H}_{2} \mathrm{O},
\end{aligned}
$$

das durch die nachfolgenden Derivate mit dem durch die katalytische Platin-Reduktion des $N$-Dimetbyl-anilins erbaltenen Reduktionsprodukte identifiziert wurde ${ }^{1}$ ).

\section{Das}

Trimethyl-cyclohexyl-ammoniumjodid, $\left(\mathrm{CH}_{3}\right)_{3} \mathrm{~N}\left(\mathrm{C}_{6} \mathrm{H}_{11}\right)$. J, schied sich beim Erkalten der erwärmten Lösung von $10 \mathrm{~g}$ Dimethylcyclohexylamin und $10 \mathrm{~g}$ Methyljodid in alkoholischer Lösung ab und krystallisierte aus Alkohol in weißen Nadeln rom Schmp. 2770

0.1054 g Sbst: : $4.9 \mathrm{ccm} \mathrm{N}\left(20^{\circ}, 746 \mathrm{~mm}\right)$.

$$
\begin{aligned}
& \mathrm{C}_{9} \mathrm{H}_{20} \mathrm{NJ} \text { (269). Ber. N 5.20, J 47.1. } \\
& \text { Gef. * 5.19, * } 46.9 \text {. }
\end{aligned}
$$

$10 \mathrm{~g}$ Trimethyl-cyclohexyl-ammoniumjodid schieden beim Schütteln mit $20 \mathrm{~g}$ Silberoxyd eiren Niederschlag von Jodsilber unter Bildung der quaternären Ammoniumbase ab, die beim Eindampfen in der wäßrigen Lösung völlig in Tetrabydro-benzol, 'Trimethylamin und Wasser zerfiel. Das überdestillierte Tetrahydro-benzol wurde isoliert und siedete bei $80-82^{\circ}$.

$$
\mathrm{C}_{6} \mathrm{H}_{11} \cdot \mathrm{N}\left(\mathrm{CH}_{3}\right)_{3} \cdot \mathrm{OH}=\mathrm{C}_{6} \mathrm{H}_{10}+\mathrm{N}\left(\mathrm{CH}_{3}\right)_{3}+\mathrm{H}_{2} \mathrm{O} \text {. }
$$

0.112 g Sbst.: $0.360 \mathrm{~g} \mathrm{CO}_{2}, 0.123 \mathrm{~g} \mathrm{H}_{2} \mathrm{O}$.

$$
\begin{aligned}
& \mathrm{C}_{6} \mathrm{H}_{30} \text { (8:). Ber. C 87.80, H 12.19. } \\
& \text { Gef. \$ 89.66, 》1\%.29. }
\end{aligned}
$$

Das Pikrat des $N$-Dimethyl-cyclohexylamins krystallisierte aus Alkohol in Stäbchen vom Schmp. 181 .

$0.2418 \mathrm{~g}$ Sbst: $33.4 \mathrm{ccm} \mathrm{N}\left(19^{\circ}, 754 \mathrm{~mm}\right)$.

$\mathrm{C}_{34} \mathrm{H}_{20} \mathrm{O}_{7} \mathrm{~N}_{4}$.(356). Ber. $\mathrm{N}$ 15.7. Gef. $\mathrm{N} 15.6$.

I) В. 52, 1527 [1919]. 
Nitroso-Verbindung. Beim Versetzen der ätherischen Lösung von $N$-Dimethyl-cyclohexylamin mit Nitrosylchlorid unter Kühlung entstand unter Abscheidung des salzsauren Dimethyl-cyclohexylamins (das aus Wasser krystallisiert bei $224^{\circ}$ schmolz) in glatter Reaktion das früher beschriebene $N-N$ itroso-N-methyl-cyclobexylamin, ein Öl rom Sdp. $121^{\circ}$ bei $12 \mathrm{~mm}$.

$0.1792 \mathrm{~g}$ Sbst.: $30.5 \mathrm{cem} \mathrm{N}\left(14^{\circ}, 742 \mathrm{~mm}\right)$.

$\mathrm{C}_{7} \mathrm{H}_{14} \mathrm{ON}_{2}$ (142). Ber. N 19.72. Gef. N 19.45.

Das

$N$-Nitroso-N-methyl-cyclobexylamin, $\mathrm{C}_{6} \mathrm{H}_{11} \cdot \mathrm{N}(\mathrm{NO}) . \mathrm{CIH}_{3}$, wurde in salzsaurer Lösung mit Zinn unter Erwärmen reduziert, das Zinn mit Schwefelwasserstoff gefältt, das Filtrat eingedampft, alkalisch gemacht und ausgeäthert. Nach dem Verdampfen des getrockneten Äthers wurde eine Base vom Sdp. $147^{\circ}$ erhalten, die auch durch ihre Benzoyl-Verbindung vom Schmp. $76^{\circ}$ als $N$-Methyl-cyclobexyla min, $\mathrm{C}_{6} \mathrm{H}_{11} \cdot \mathrm{NH} . \mathrm{CH}_{3}$, charakterisiert wurde.

Bei Einwirkung von Nitrosylchlorid auf die trockne ätherische Lösung von $\mathrm{N}$-Methyl-dicyclohexylamin $\left.{ }^{1}\right), \mathrm{C}_{6} \mathrm{H}_{11} . \mathrm{N}\left(\mathrm{CH}_{3}\right) . \mathrm{C}_{6} \mathrm{H}_{11}$, die unter starkem Geruch nach Formaldehyd erfolgte, bildeten sich aus der ausgeätherten Lösung des Reaktionsproduktes Krystalle rom Schmp. $105^{\circ}$, die $N$-Nitroso-Verbindung des Dicyclohexylamins, $\mathrm{C}_{6} \mathrm{H}_{11} . \mathrm{N}(\mathrm{NO}) . \mathrm{C}_{6} \mathrm{H}_{11}$.

Diese Nitroso-Verbindung wurde der Reduktion mit Zink und Salzsäure unterworfen und die erhaltene Base aus dem alkalisch gemachten Reaktionsprodukt mit Wasserdämpfen destilliert. Sie erwies sich durch ihren Sdp. 250 und den Scbmp. 20 als Dicyclobexylamin, $\mathrm{C}_{6} \mathrm{H}_{11} . \mathrm{NH} . \mathrm{C}_{6} \mathrm{H}_{11}$. Diese Base wurde noch durch katalytische Reduktion des Diphenylamins mit kolloidem Platin hergestellt und dureb ibre Nitroso-V́erbindung rom Schmp. $105^{\circ}$ identifiziert.

\section{Mit Jodäthyl.}

a) Zu N-Äthyl-cyclohexyla min. Unter Eiskühlung reagierten die beiden Komponenten glatt zu dem jodwasserstoffsauren Salz. Beim Schütteln mit Benzoylchlorid in alkalischer Lösung ging alles in die Benzoyl-Verbindung äber, die bei $201^{\circ}$ siedete. Diese Benzoglverbindung erstarrte nach längerem Stehen zu einem Krystallbrei.

$0.1634 \mathrm{~g}$ Sbst.: $8.5 \mathrm{ccm} \mathrm{N}\left(13^{0}, 742 \mathrm{~mm}\right)$.

$\mathrm{C}_{15} \mathrm{H}_{21} \mathrm{ON}$ (231). Ber. N 6.06. Gef. N 5.97.

Die durch alkoholisches Kali verseifte Base erwies sich durch ibren Siedepunkt, sowie durch nachfolgend beschriebene Derivate als

1) A. Skita und W. Berendt, B. 52, 1522 [1919]. 
identisch mit dem durch die katalytische Platin-Reduktion des $N$-Äthyl-anilins erhaltenen $N$-Äthyl-cyclohexylamin ${ }^{2}$ ).

Acetyl-Verbindung: Öl rom Sdp. $256^{\circ}$ bei $740 \mathrm{~mm}$.

$0.2372 \mathrm{~g}$ Shst.: $18.2 \mathrm{ccm} \mathrm{N}\left(21^{0}, 742 \mathrm{~mm}\right)$.

$$
\mathrm{C}_{10} \mathrm{H}_{19} \mathrm{ON} \text { (169). Ber. N 8.3. Gef. N 8.4. }
$$

Nitroso-Verbindung: (̈) vom Sdp. $130^{\circ}$ bei $12 \mathrm{~mm}$, erhalten durch Einwirkung von Nitrosylchlorid auf die Base in ätherischer Lösung, wobei sich das salzsaure Salz des $N$ - ̈̈tbyl-cyclohexylamins abschied, das in Krystallen aus Wasser rom Schmp. $184^{\circ}$ erhalten wurde.

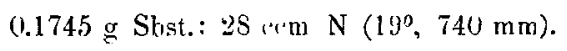

$\mathrm{C}_{8} \mathrm{H}_{16} \mathrm{ON}_{2}$ (156). Ber. N 17.9. Get. N 17.8.

Pikrat: Gelbe Krystallo aus Alkohol vom Schmp. $133^{\circ}$.

$0.2420 \mathrm{~g}$ Sbst.: $3.6 \mathrm{~cm} \mathrm{~N}\left(19^{0}, 754 \mathrm{~mm}\right)$.

$\mathrm{C}_{14} \mathrm{H}_{20} \mathrm{O}_{7} \mathrm{~N}_{4}$ (356). Ber. $\mathrm{N}$ 15.7. Gef. $\mathrm{N} 15.8$.

b) Zu N-Diäthyl-cyclohexylamin.

Aquimolekulare Mengen Jodätbyl und Cyclobexylanin, mit der berechneten Menge Kali am Rückflußkühler gekocbt, gaben nach dem Alkalischmachen des Reaktionsproduktes eine Base vom einheitlichen Sdp. $193^{\circ}$, die sich mit dem durch die katalytische Platin-Reduktion des $N$-Dimethyl-anilins erhaltenen $N$-Diäthyl-cyclohexylamin ${ }^{2}$ ) durch gleichen Siedepunkt, sowie durch gleiche Derivate identifizieren ließ. Das salzsaure Salz schmolz oberhalb $230^{\circ}$, das Pikrat krystallisierte aus Alkobol in gelben Platten vom Schmp. $98^{\circ}$.

0.3000 g Sbst.: $39.0 \mathrm{~cm} \mathrm{~N}\left(20^{\circ}, 752 \mathrm{~mm}\right)$.

$\mathrm{C}_{16} \mathrm{H}_{24} \mathrm{O}_{7} \mathrm{~N}_{4}$ (984). Ber. N 14.5. Gef. N 14.6 .

\section{Die Platin-Katalyse des Antifebrins und seiner Homologen.}

Während das Acetanilid in denselben Mengenverbältnissen, in denen die Lmwandlung des Anilins in Cyclohexylamin vorgenommen wurde, der Aufnahme ron Wasserstofi Widerstaud entgegensetzte, konnte es bei größerer Platin-Konzentration und einer Temperatur von $70-80^{\circ}$ in $12-16 \mathrm{Stdn}$ völlig in das Acetyl-cyclohexylamin rom Scbmp. $107^{\circ}$ umgewandelt werden.

Bei weiterer Konzehtration gelang es schließlich - obne die Platinmenge $z$ u erhöhen ${ }^{3}$ ) --, sowohl das Acetavilid, als auch die bomologen Acetyl-o-, $-m-$ u. - $p$-toluidine innerhalb 4j Minuten glatt in die korrespondierenden acetylierten Cyclohexylamine umzuwandeln.
1) lor. cit.
$\Rightarrow$ lnc. eit.
3) 2. 52, $1529[1919]$. 
$9.1 \mathrm{~g}$ Acetanilid bezw. $10 \mathrm{~g} \mathrm{or}, m$-oder $p$-Acettoluid wurden in einer Lösung von $15 \mathrm{ccm}$ einer 10-proz. Platinchlorwasserstoffsäure (enthaltend $0.7 \mathrm{~g} \mathrm{Pt}$ ), $50 \mathrm{ccm}$ kolloider Platinlösung (enthaltend $0.15 \mathrm{~g}$ Platin), $20 \mathrm{ccm}$ 10-proz. Gummi-arabicum-Lösung und $27 \mathrm{ccm}$ Wasser suspendiert und bei $70-85^{\circ}$ mit Wasserstoff unter $3 \mathrm{Atm}$. Überdruck geschüttelt. Nachdem die theoretische Menge in 45 Minuten aufgenommen und eine weitere Absorption nicht mehr erfolgt war, wurden die Acetylverbindungen nach dem Eindampien des Reaktionsproduktes zur Trockne durch Schütteln mit Äther aufgenommen, im Vakuum destilliert und aus Alkohol krystallisiert.

Acetyl-2-methyl-cyclohexylamin, Sdp.20 154 , Schmp. 50

$0.2345 \mathrm{~g}$ Sbst.: $18.2 \mathrm{ccm} \mathrm{N}\left(13^{\circ}, 740 \mathrm{~mm}\right)$.

$\mathrm{C}_{9} \mathrm{H}_{17} \mathrm{ON}$ (155). Ber. N 9.03. Gef. N 9.01.

Acetyl-3-methyl-cyelohexylamin, Sdp.18 $152^{\circ}$, Schmp. $74-75^{\circ}$.

$0.2678 \mathrm{~g}$ Sbst.: $20.75 \mathrm{ccm} \mathrm{N}\left(13^{0}, 740 \mathrm{~mm}\right)$.

$\mathrm{C}_{9} \mathrm{H}_{17} \mathrm{ON}(155)$. Ber. N 9.03. Gef. N 9.00 .

Acetyl-4-methyl-cyclohexylamin, Sdp.12 1520, Schmp. 77-780.

$0.2745 \mathrm{~g}$ Sbst.: $21.1 \mathrm{ccm} \mathrm{N}\left(13^{\circ}, 740 \mathrm{~mm}\right)$.

$\mathrm{C}_{9} \mathrm{H}_{37} \mathrm{ON}$ (155). Ber. N 9.03. Gef. N 8.93 .

An freien Cyclohexylaminen waren keine wägbaren Mengen entstariden, und die Acetyl-Verbindungen wurden in so gut wie quantitativen Ausbeuten erhalten.

V. Über die katalytiscbe Reduktion von Amino-phenolen:

1. Des Phenacetins zu Hexahydro-antifebrin.

$17.9 \mathrm{~g}$ Phenacetin, gelöst in $100 \mathrm{ccm}$ Eisessig, wurden mit $21 \mathrm{ccm}$ einer 10-proz. Platinchlorwasserstoffsäure (enthaltend $1 \mathrm{~g}$ Platin), $15 \mathrm{ccm}$ kolloider Platinlösung (enthaltend $0.045 \mathrm{~g} \mathrm{Pt}$ ), $2 \mathrm{~g}$ Gummi arabicum und $65 \mathrm{ccm}$ Wasser versetzt und bei $75^{\circ}$ unter $3 \mathrm{Atm}$. ÜLerdruck geschüttelt. Die Wasserstoff -A ufuahme war bereits in $1 \frac{1}{2}$ Stdn. beendet und betrug $9.0 \mathrm{l}$, entsprechend $4 \mathrm{Mol}$. Das Reaktionsprodukt wurde im Vakuum zur Trockne gedampit und in absolutem Alkohol aufgenommen; es krystallisierte in schönen weißen Nadeln rom Schmp. 106-1070, erwies. sich also als Acetyl-cyclohexylamin.

$0.1005 \mathrm{~g}$ Sbst.: $0.2516 \mathrm{~g} \mathrm{CO}_{2}, 0.0955 \mathrm{~g} \mathrm{H}_{2} \mathrm{O} .-0.0772 \mathrm{~g}$ Sbst.: $7.0 \mathrm{ccm}$ $\mathrm{N}\left(17^{0}, 742 \mathrm{~mm}\right)$.

$$
\begin{aligned}
& \mathrm{C}_{8} \mathrm{H}_{15} \mathrm{ON} \text { (141). Ber. C 68.10, H 10.64, N 9.94. } \\
& \text { Gef. • 68.28, "10.67, " } 10.1 \% \text {. }
\end{aligned}
$$

Anisol und Phenetol gaben unter diesen Bedingungen bei der Reduktion mit kolloidem Platin quantitativ den Cyclobexyl-methylbezw. -äthyl-äther, wobei die Reduktionsprodukte aus der alkalisch gewiachten Lösung mit Wasserdampf entfernt wurden.

Berichte d. D. Chem. Oesellschaft. Jahrg. LIII. 
Hexahydro-anisol, Sdp. $140^{\circ}$ bei $740 \mathrm{~mm}$.

0.1986 g Sbst.: $0.5376 \mathrm{~g} \mathrm{CO}_{2}, 0.2128 \mathrm{~g} \mathrm{H}_{2} \mathrm{O}$.

$$
\mathrm{C}_{7} \mathrm{H}_{14} \mathrm{O} \text { (114). Ber. C 73.68, } \mathrm{H} 12.28 \text {. }
$$

Gef. - 73.82, 12.00 .

Hexahydro-phenetol, Sdp. $144-145^{\circ}$ bei $738 \mathrm{~mm}$.

$0.1347 \mathrm{~g}$ Sbst.: $0.3710 \mathrm{~g} \mathrm{CO}_{2}, 0.1521 \mathrm{~g} \mathrm{H}_{2} \mathrm{O}$.

$$
\begin{aligned}
& \mathrm{C}_{8} \mathrm{H}_{16} \mathrm{O} \text { (128). Ber. C 75.00, H } 12.50 \text {. } \\
& \text { Gef. 》75.11, 》12.63. }
\end{aligned}
$$

2. Des Anisidins und Phenetidins zu Cyclohexylamin.

$12.3 \mathrm{~g}$ Anisidin bezw. $13.7 \mathrm{~g}$ Phenetidin wurden in eine Mischung von $50 \mathrm{ccm}$ Eisessig und $5 \mathrm{ccm}$ Salzsäure (spez. Gew. 1.19) eingetragen, mit einer Lösung von $21 \mathrm{ccm}$ 10-proz. Platinchlorwasserstoffsäure (enthaltend $1 \mathrm{~g}$ Platin), $30 \mathrm{ccm}$ kolloider Platinlösung (enthaltend $0.09 \mathrm{~g} \mathrm{Pt}$ ), $2 \mathrm{~g}$ Gummi arabicum in $75 \mathrm{ccm}$ Wasser versetzt und bei $40-50^{\circ}$ mit Wasserstolf unter $3 \mathrm{Atm}$. Überdruck geschüttelt. Die Absorption von $4 \mathrm{Mol}$. war in beiden Fällen in 3 Stdn. beendet. Aus der alkalisch gemacbten Reaktionsflüssigkeit destillierte in beiden Fällen ein Basengemisch über, das sich in eine Base vom Sdp. 134 $-135^{\circ}$ und eine rom Sdp.30 $145^{\circ}$ zerlegen ließ. Erstere wurde durch ihre Acetylverbindung vom Schmp. $107^{\circ}$ als Cyclobexylamin erkanot; letztere erwies sich auch durch den Schmelzpunkt $\left(330^{\circ}\right)$ ibres salzsauren Salzes als Dicyclohexylamin.

Zur raschen Identifizierung besonders kleiner Mengen Cyclohexylamins ist das Toluol-p-sulfonsäure-Derivat, $\mathrm{CH}_{3} \cdot \mathrm{C}_{6} \mathrm{H}_{4} . \mathrm{SO}_{2}$. NH. $\mathrm{C}_{6} \mathrm{H}_{11}$, besonders geeignet. $2 \mathrm{~g}$ Cyclohexylamin wurden mit $17 \mathrm{ccm}$ 10-proz. Natronlauge und $4 \mathrm{~g}$ Toluol-p-sulfonsäurechlorid versetzt und erwärmt, wobei sich ein beim Erkalten erstarrendes Öl abschied. Krystalle aus Weingeist vom Schmp. $86^{\circ}$.

$0.4469 \mathrm{~g}$ Sbst.: $0.4004 \mathrm{~g} \mathrm{BaSO}$.

$$
\mathrm{C}_{13} \mathrm{H}_{19} \mathrm{O}_{2} \mathrm{NS} \text { (253). Ber. S 12.6. Gef. S 12.3. }
$$

3. Des $p$ - und $m$-Amino-phenols zu Cyclohexylamin.

$12 \mathrm{~g} p$ - bezw. $m$-Amino-phenol, gelöst in $50 \mathrm{ccm}$ Wasser und $8 \mathrm{~g}$ Salzsäure (spez. Gew. 1.19), wurden mit einer Lösung von $21 \mathrm{ccm}$ 10-proz. Platinchlorwasserstoffsäure (enthaltend $1 \mathrm{~g} \mathrm{Pt}), 30 \mathrm{ccm}$ kolloider Platinlösung (enthaltend $0.09 \mathrm{~g} \mathrm{Pt}$ ), $2 \mathrm{~g}$ Gummi arabicum, $50 \mathrm{ccm}$ Eisessig, $90 \mathrm{ccm}$ Wasser versetzt und bei $60-70^{\circ}$ mit Wasserstoff unter $3 \mathrm{Atm}$. Überdruck geschüttelt, wobei in $4 \mathrm{Stdn}$. die für 4 Mol. berechnete Menge Wasserstoff absorbiert war. Auch hier war ein Gemisch von Cyclohexylamin und Dicyclohexylamin entstanden, wobei letzteres sich in größerer Menge gebildet hatte und noch durch die Analyse seines salzsauren Salzes vom Schmp. ?300 bestimmt wurde. 
$0.1337 \mathrm{~g}$ Sbst.: $8.0 \mathrm{cem} \mathrm{N}\left(17^{0}, 732 \mathrm{~mm}\right) .-0.1297 \mathrm{~g}$ Sbst.: $6.03 \mathrm{ccm}$ ${ }^{n} / 10-\mathrm{AgNO}$.

$$
\begin{array}{r}
\mathrm{C}_{12} \mathrm{H}_{24} \mathrm{NCl}(217) . \quad \text { Ber. } \mathrm{N} 6.43 \text {, Cl 16.32. } \\
\\
\text { Gef. } \$ 6.65 \text {, 》 16.48. }
\end{array}
$$

4. Vono-Amino-phenol zu Dodekabydro-phenoxazin (S. 1246).

Ein Gemisch von $15 \mathrm{~g}$ o-Nitro-phenol, $50 \mathrm{ccm}$ Eisessig, $50 \mathrm{ccm}$ Wasser, 8 g Salzsäure (spez. Gew. 1.19), 21 ccm 10-proz. Platinchlorwasserstoffsäure (enthaltend $1 \mathrm{~g} \mathrm{Pt}$ ), $20 \mathrm{ccm}$ kolloider Platinlösung (enthaltend $0.06 \mathrm{~g} \mathrm{Pt}$ ) und $2 \mathrm{~g}$ Gummi arabicum wurden mit Wasserstoff unter $3 \mathrm{Atm}$. Überdruck geschüttelt, worauf unter rapider $\mathbf{A b}$ sorption und Erwärmung zunächst die Reduktion der Nitrogruppe erfolgte und sich eine klare kolloide Lösung bildete. Die Hydrierung des Benzolkerns wurde bei $70^{\circ}$ durchgeführt und war in 2 Stdn. beendet. Aus der alkalisch gemachten Reaktionsflüssigkeit destillierte zunächst Cyclohexylamin und sodann ein im Destillat erstarrender Körper über, der aus Petroläther Krystalle vom Schmp. 103-104" lieferte.

$0.0736 \mathrm{~g}$ Sbst.: $0.1985 \mathrm{~g} \mathrm{CO}_{3}, 0.0700 \mathrm{~g} \mathrm{H}_{2} \mathrm{O} .-0.0910 \mathrm{~g}$ Sbst.: $6.2 \mathrm{cem}$ $\mathrm{N}\left(20^{\circ}, 732 \mathrm{~mm}\right)$.

$$
\begin{aligned}
& \mathrm{C}_{12} \mathrm{H}_{21} \mathrm{ON} \text { (195). Ber. C 73.85, H 10.77, N 7.19. } \\
& \text { Gef. > 73.06, } 10.65,>7.46 .
\end{aligned}
$$

Die nach Schotten-Baumann hergestellte Benzoyl-Verbindung krystallisierte aus Alkohol in Pyramiden vom Schmp. 160-161 ${ }^{\circ}$. $0.1041 \mathrm{~g}$ Sbst.: $4.1 \mathrm{ccm} N\left(18^{\circ}, 742 \mathrm{~mm}\right)$.

$\mathrm{C}_{19} \mathrm{H}_{23} \mathrm{O}_{2} \mathrm{~N}$ (299). Ber. $\mathrm{N}$ 4.68. Gef. $\mathrm{N} 4.42$.

140. Karl Fleischer und Fritz Siefert:

Ưber Abkömmlinge des ac.-Tetrahydro-naphthalins und Tetrahydro-acenaphthens.

[Aus dem Chemischen Thnśtitut der Universität Frankfurt a. M.]

(Eingegangen am 12. Mai 1920.)

Über die Einwirkung substituierter Malonsäurechloride auf partiell hydrierte aromatische Kohlenwasserstoffe, wie Tetrahydro-naphthalin, Tetrahydro-acenaphthen und Tetrahydro-diphenyl, hat der eine von uns (F.) bereits am 24. Juli 1919 und am 24. Februar 1920 in der Frankfurter Chemischen Gesellschaft berichtet ${ }^{1}$ ). Veranlaßt durch das zweite, unten genannte Referat batte Hr. Prof.

1) Z. Ang. 32, II 596 [1919]; 33, II 160 [1920]. Ch. Z. 44, 464 [1920]. 